\title{
Dual function synergists based on glucose and sucrose for gas hydrate and corrosion inhibition
}

Abdolreza Farhadian, ${ }^{\mathrm{a}, \mathrm{b}, \mathrm{c}}$ Mikhail A. Varfolomeev, ${ }^{\mathrm{a}, \mathrm{b},{ }^{*}}$ Anton P. Semenov, ${ }^{\mathrm{d}}$ Rais I. Mendgaziev, Andrey S. Stoporev, b,d,e

a Department of Petroleum Engineering, Kazan Federal University, Kremlevskaya str. 18, 420008 Kazan, Russian Federation

b Department of Physical Chemistry, Kazan Federal University, Kremlevskaya str. 18, 420008

Kazan, Russian Federation

${ }^{c}$ Research Center for Oil and Gas Exploration, Shahid Beheshti University, GC, 1983969411, Tehran, Iran

d Gubkin University, Department of Physical and Colloid Chemistry, 65, Leninsky prospekt, Building 1, Moscow 119991, Russian Federation

e Novosibirsk State University, Pirogova street 1, Novosibirsk 630090, Russian Federation

* Corresponding author E-mail: mikhail.varfolomeev@kpfu.ru 


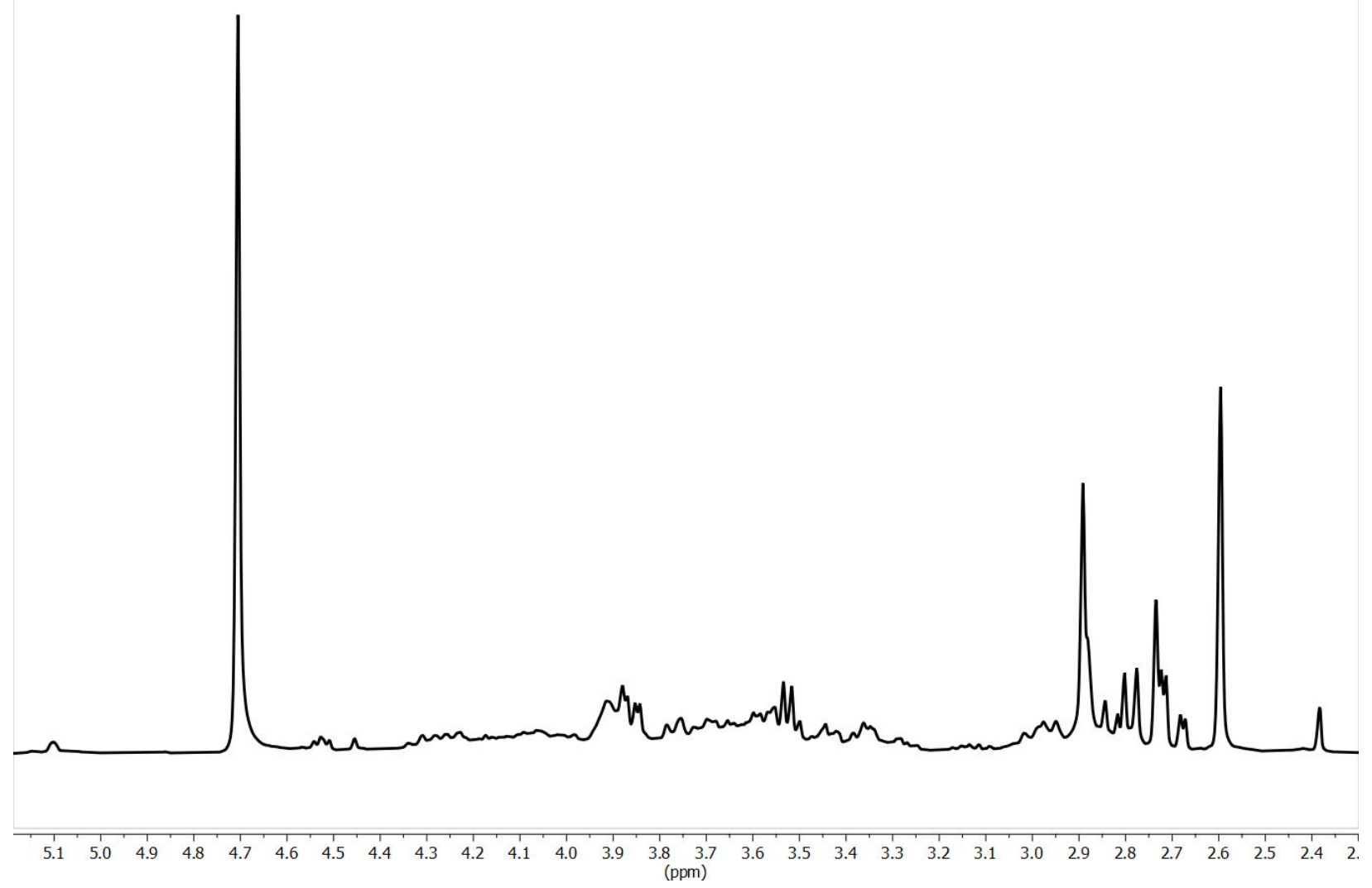

Figure S1. ${ }^{1} \mathrm{H}$ NMR spectrum of SBS. 


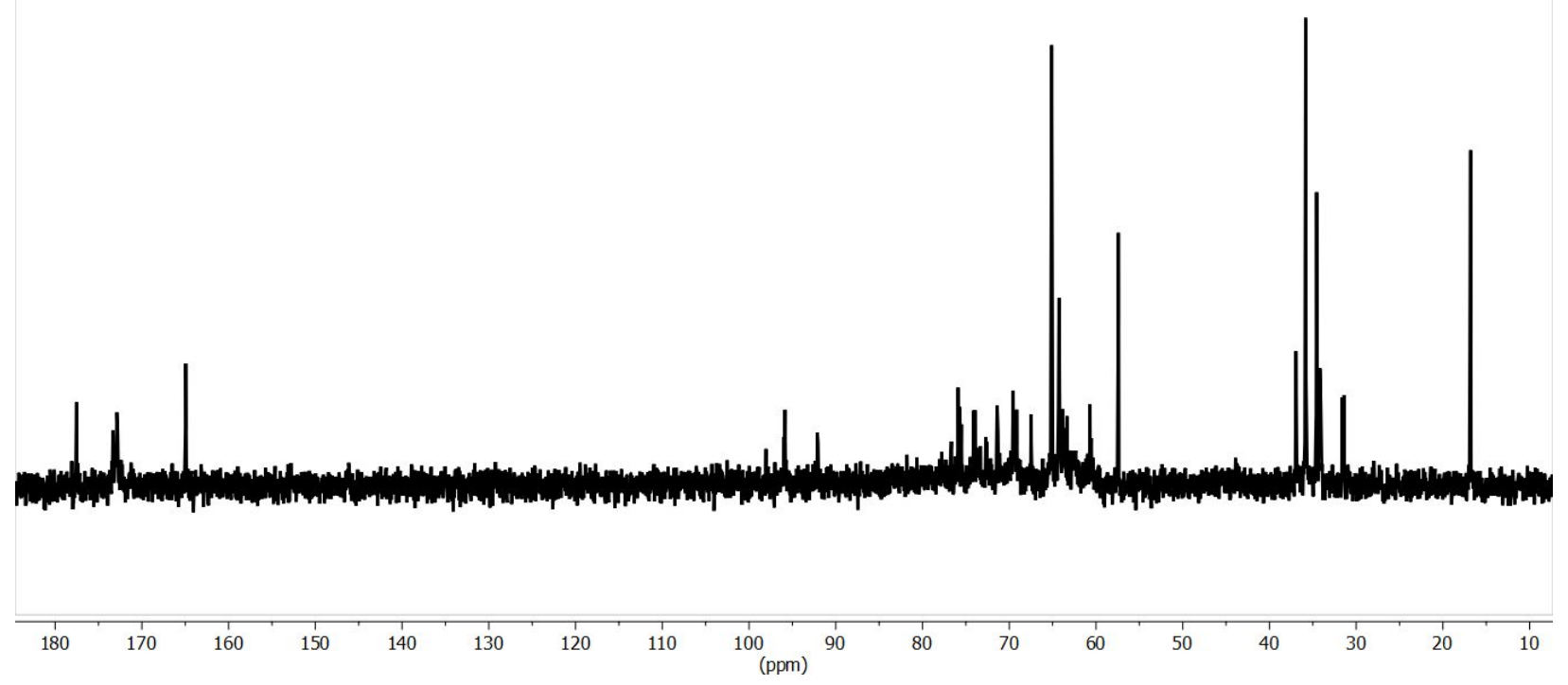

Figure S2. ${ }^{13} \mathrm{C}$ NMR spectrum of SBS. 


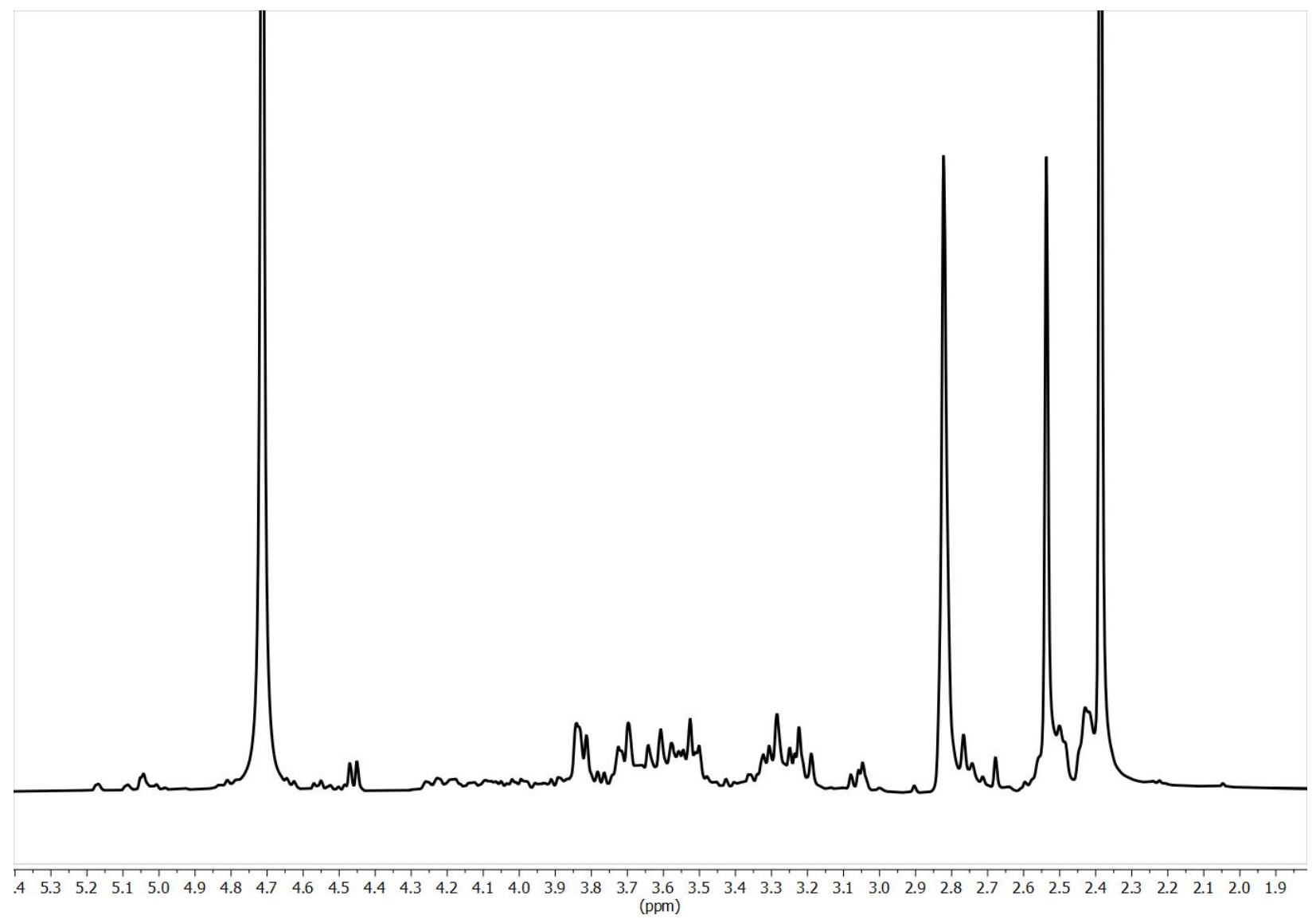

Figure S3. ${ }^{1} \mathrm{H}$ NMR spectrum of GBS. 


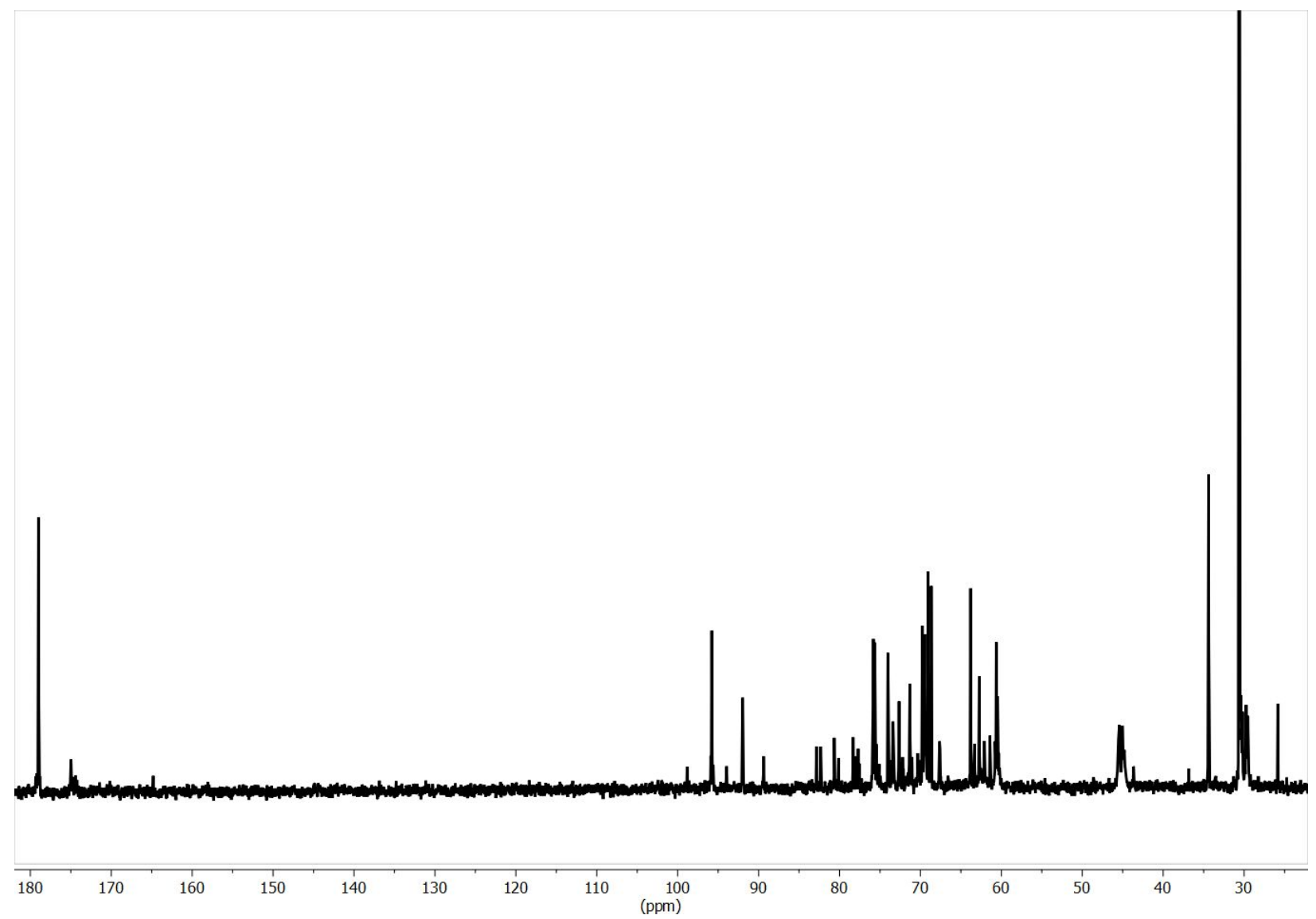

Figure S4. ${ }^{13} \mathrm{C}$ NMR spectrum of GBS. 

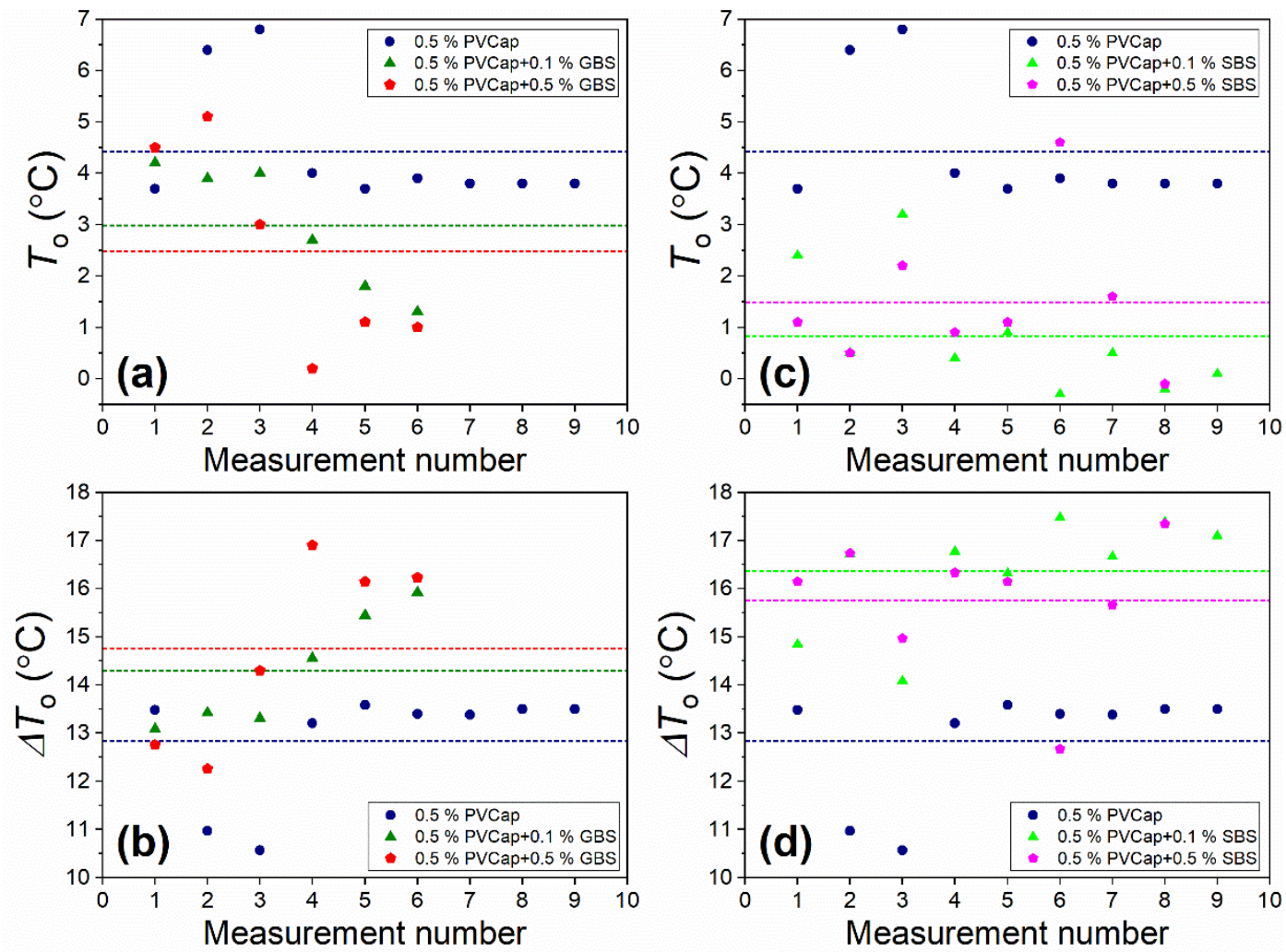

Figure S5. Hydrate onset temperatures and subcoolings vs. measurement number for the polymer PVCap and its mixtures with GBS and SBS; horizontal color dashed line shows mean value for each sample. 

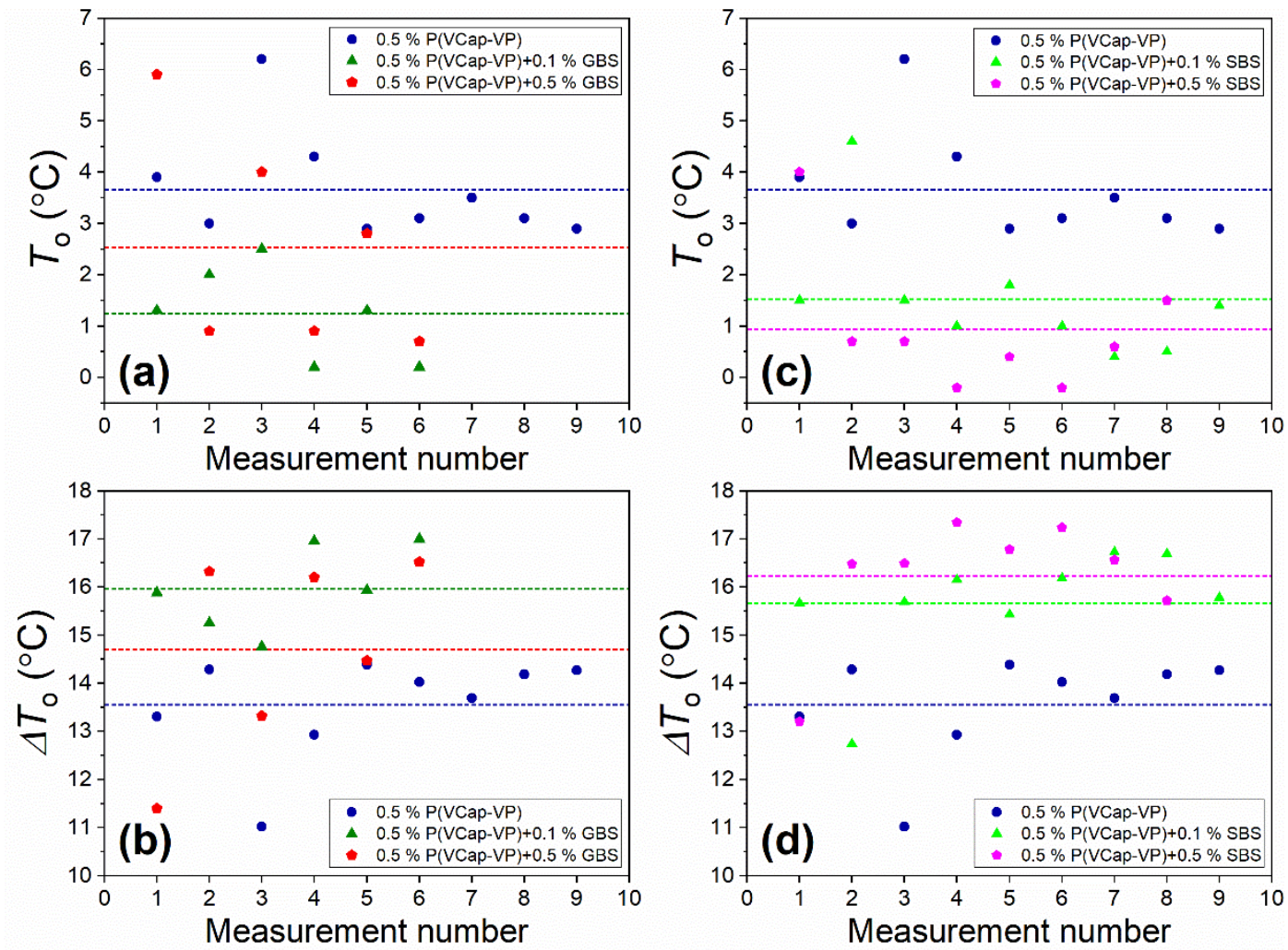

Figure S6. Hydrate onset temperatures and subcoolings vs. measurement number for the copolymer P(VCap-VP) and its mixtures with GBS and SBS; horizontal color dashed line shows mean value for each sample. 
Table S1. Statistical significance between the mean values of hydrate onset subcooling for the samples with and without synergists for $0.5 \%$ PVCap and mixed samples.

\begin{tabular}{|l|l|l|l|l|l|}
\hline \multirow{2}{*}{ Sample } & \multicolumn{5}{|c|}{ Test / Significance (Probability) } \\
\cline { 2 - 6 } & Bonferroni & Scheffe & Tukey & Sidak & Fisher \\
\hline PVCap/GBS 0.5/0.1 & $\mathbf{0}(0.2206)$ & $\mathbf{0}(0.1929)$ & $\mathbf{0}(0.1673)$ & $\mathbf{0}(0.2048)$ & $\mathbf{0}(0.0735)$ \\
\hline PVCap/GBS 0.5/0.5 & $\mathbf{0}(0.0634)$ & $\mathbf{0}(0.0652)$ & $\mathbf{0}(0.0527)$ & $\mathbf{0}(0.0621)$ & $\mathbf{1}(0.0212)$ \\
\hline PVCap/SBS 0.5/0.1 & $\mathbf{1}\left(1.3 \cdot 10^{-5}\right)$ & $\mathbf{1}\left(2.1 \cdot 10^{-5}\right)$ & $\mathbf{1}\left(1.2 \cdot 10^{-5}\right)$ & $\mathbf{1}\left(1.3 \cdot 10^{-5}\right)$ & $\mathbf{1}\left(4.3 \cdot 10^{-6}\right)$ \\
\hline PVCap/SBS $0.5 / 0.5$ & $\mathbf{1}\left(2.5 \cdot 10^{-4}\right)$ & $\mathbf{1}\left(3.7 \cdot 10^{-4}\right)$ & $\mathbf{1}\left(2.3 \cdot 10^{-4}\right)$ & $\mathbf{1}\left(2.5 \cdot 10^{-4}\right)$ & $\mathbf{1}\left(8.2 \cdot 10^{-5}\right)$ \\
\hline
\end{tabular}

0 indicates that the difference of the means between pure and mixed sample is insignificant

1 indicates that the difference of the means between pure and mixed sample is significant

Table S2. Statistical significance between the mean values of hydrate onset subcooling for the samples with and without synergists for $0.5 \% \mathrm{P}(\mathrm{VCap}-\mathrm{VP})$ and mixed samples.

\begin{tabular}{|l|l|l|l|l|l|}
\hline \multirow{2}{*}{ Sample } & \multicolumn{5}{|c|}{ Test / Significance (Probability) } \\
\cline { 2 - 6 } & Bonferroni & Scheffe & Tukey & Sidak & Fisher \\
\hline P(VCap-VP)/GBS 0.5/0.1 & $\mathbf{1}(0.0121)$ & $\mathbf{1}(0.0143)$ & $\mathbf{1}(0.0107)$ & $\mathbf{1}(0.0121)$ & $\mathbf{1}(0.0040)$ \\
\hline $\mathrm{P}($ VCap-VP)/GBS 0.5/0.5 & $\mathbf{0}(0.4049)$ & $\mathbf{0}(0.3172)$ & $\mathbf{0}(0.2857)$ & $\mathbf{0}(0.3527)$ & $\mathbf{0}(0.1350)$ \\
\hline $\mathrm{P}($ VCap-VP)/SBS 0.5/0.1 & $\mathbf{1}(0.0032)$ & $\mathbf{1}(0.0042)$ & $\mathbf{1}(0.0029)$ & $\mathbf{1}(0.0032)$ & $\mathbf{1}(0.0011)$ \\
\hline $\mathrm{P}($ VCap-VP)/SBS 0.5/0.5 & $\mathbf{1}\left(3.9 \cdot 10^{-4}\right)$ & $\mathbf{1}\left(5.7 \cdot 10^{-4}\right)$ & $\mathbf{1}\left(3.7 \cdot 10^{-4}\right)$ & $\mathbf{1}\left(3.9 \cdot 10^{-4}\right)$ & $\mathbf{1}\left(1.3 \cdot 10^{-4}\right)$ \\
\hline
\end{tabular}

0 indicates that the difference of the means between pure and mixed sample is insignificant

1 indicates that the difference of the means between pure and mixed sample is significant 